\title{
Coexistence of collapse and stable spatiotemporal solitons in multimode fibers
}

\author{
Olga V. Shtyrina, ${ }^{1,2}$ Mikhail P. Fedoruk,,${ }^{1,2}$ Yuri S. Kivshar, ${ }^{3}$ and Sergei K. Turitsyn ${ }^{1,4}$ \\ ${ }^{1}$ Novosibirsk State University, Novosibirsk 630090, Russia \\ ${ }^{2}$ Institute of Computational Technologies SB RAS, Novosibirsk 630090, Russia \\ ${ }^{3}$ Nonlinear Physics Center, Research School of Physics and Engineering, Australian National University, Canberra ACT 2601, Australia \\ ${ }^{4}$ Aston Institute of Photonics Technologies, Aston University, Birmingham B4 7ET, England, United Kingdom
}

(Received 24 October 2016; revised manuscript received 7 September 2017; published 25 January 2018)

\begin{abstract}
We analyze spatiotemporal solitons in multimode optical fibers and demonstrate the existence of stable solitons, in a sharp contrast to earlier predictions of collapse of multidimensional solitons in three-dimensional media. We discuss the coexistence of blow-up solutions and collapse stabilization by a low-dimensional external potential in graded-index media, and also predict the existence of stable higher-order nonlinear waves such as dipole-mode spatiotemporal solitons. To support the main conclusions of our numerical studies we employ a variational approach and derive analytically the stability criterion for input powers for the collapse stabilization.
\end{abstract}

DOI: 10.1103/PhysRevA.97.013841

Optical solitons [1] are usually associated with lowdimensional nonlinear systems such as single-mode optical fibers or planar waveguides, and their existence in higherdimensional systems in the form of spatiotemporal localized waves is relatively rare, and it requires strong nonlinearity saturation [1], spatial nonlocality [2], or other physical mechanisms arresting wave collapse [3,4]. Wave collapse (also known as blow-up or self-focusing) occurs in a range of physical systems including nonlinear optics, plasmas, fluid dynamics, physics of atmosphere and ocean, and solid-state physics [5]. Typically, wave collapse is associated with multidimensional physical problems [3-13]. From a broader perspective, wave collapse is the process of the singularity formation in a finite time (or at a finite distance), which is typically arrested by higher-order effects not accounted for in the original model. Effect of wave collapse can be exploited for compression of optical pulses [8-11] and optical pulse fusion [11,12]. An arrest of wave collapse and emergence of stable coherent structures in higher-dimensional systems have been studied in various physical contexts (see, e.g., the review paper [14] and references therein). Solitons localized in time and one transverse spatial dimension have been observed in quadratic media [15], and such solitons suffer from modulation instability which breaks elliptical beams into filaments. Threedimensional spatiotemporal solitons were demonstrated in arrays of weakly coupled optical waveguides [16,17], but such solitons are largely controlled by the lattice discreteness being stable for a weak coupling.

For a long time, the use of single-mode optical fibers was the solution of choice for long-haul communication systems, allowing one to avoid spatial scattering of light for delivering optical signals without spatial-mode dispersion over thousands of kilometers. However, fast-growing demands on capacity of fiber systems and challenges imposed by nonlinear signal interaction attracted recent attention to the technology of spatial-division multiplexing (SDM) for future high-capacity optical communications (see, e.g., Refs. [18,19] and references therein). A solution based on the use of multiple systems over parallel fibers, while always possible, is not attractive due to linearly scaled (with growing capacity) transmission costs and power consumption. Potentially, the SDM technology might offer a cost-per-bit reduction and improved energy efficiency. One of the considered possibilities for implementing the SDM technology is the use of multimode fibers (MMFs) for parallel communication channels. In MMFs optical pathways are defined by different spatial modes, and spatial signal processing is required to separate channels at a receiver. Due to highly important SDM applications, MMFs attracted a flurry of renewed interest recently. The MMFs with large core can potentially be used for rather different albeit important high-power applications. However, a similar challenge in this case is to control the spatial coherence and resulting beam size.

Recent studies of MMFs suggest that interesting dynamics can occur in the nonlinear regime [20-29]. In this regime, the waveguide modes, which may number from a few to up to thousands, strongly affect each other through nonlinear processes $[20,25]$. The output spatial and temporal properties of light are defined by nonlinear interactions of optical paths corresponding to different spatial modes in MMFs. In general, these different paths through the MMF medium interfere, leading to a spatial speckle pattern. The different time delays corresponding to different spatial modes lead to spatial-mode dispersion and temporal distortion of pulses. However, nonlinear effects produce a spatiotemporal coherence in the propagating light, leading to new interesting possibilities.

In the graded-index MMFs an effective (transverse) parabolic potential provides the stabilization mechanism for spatiotemporal pulses. Up to now, the spatially localized structures in multidimensional trapping potentials have been analyzed only in the application to the Bose-Einstein condensates. In particular, it was shown that solitons can be stabilized by both three-dimensional parabolic $[30,32-38]$ and periodic [31] potentials. Wave collapse and coexistence of collapsing and stable multidimensional solutions in Bose-Einstein was 


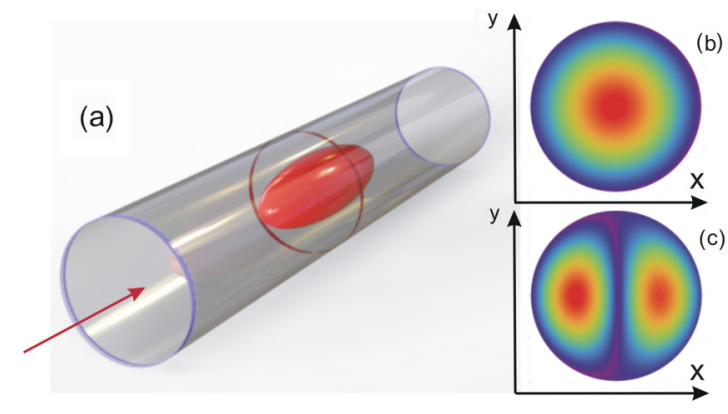

FIG. 1. Schematic illustration of stable spatiotemporal solitons propagating in a graded-index optical fiber. Two types of spatial cross-section profiles are shown on the right, namely, fundamental and dipole-mode solitons, respectively.

discussed in Refs. [13,32-35]. However, in the MMFs the waveguide forms a two-dimensional (transversal) potential in the $2+1+1$ nonlinear system. Thus, in mathematical terms, the importance of our paper is in the study of the stabilizing effect of two-dimensional parabolic potential on nonlinear dynamics of coherent structures in a three-dimensional nonlinear dispersive medium.

In this paper, we analyze stability of spatiotemporal solitons in multimode optical fibers in graded-index waveguides and demonstrate the existence of stable soliton families, as well as stable dipole-mode spatiotemporal solitons [both illustrated in Figs. 1(a)-1(c)], in similarity to higher-order localized modes in saturable media [39] and recently observed fundamental modes of multimode fibers [40].

\section{MATHEMATICAL MODEL}

Pulse propagation in a multimode graded-index optical fiber is described in the paraxial approximation by the standard nonlinear Schrödinger equation (NLSE) derived for the slowly varying pulse envelope (that includes all modes):

$$
\begin{aligned}
i \frac{\partial \psi}{\partial Z}= & \frac{\beta_{2}}{2} \frac{\partial^{2} \psi}{\partial T^{2}}-\frac{1}{2 k_{0}}\left(\frac{\partial^{2} \psi}{\partial x^{\prime 2}}+\frac{\partial^{2} \psi}{\partial y^{\prime 2}}\right) \\
& +U\left(x^{\prime}, y^{\prime}\right) \psi-\gamma|\psi|^{2} \psi
\end{aligned}
$$

where $k_{0}=\omega_{0} n_{0} / c$ is the wave number at the central frequency $\omega_{0}, \beta_{2}\left[\mathrm{fs}^{2} / \mathrm{mm}\right]$ is the group-velocity dispersion and $\gamma[\mathrm{m} / \mathrm{W}]$ is the nonlinear coefficient, and $\psi$ is the slowly varying envelope at the center frequency $\omega_{0}$ with time $T$ in the reference frame moving at the group velocity of the pulse. The effective potential $U\left(x^{\prime}, y^{\prime}\right)$ describes a variation of the refractive index that forms a mode structure in the linear propagation regime. In what follows, we consider $U\left(x^{\prime}, y^{\prime}\right)=\left(k_{0} \Delta / R^{2}\right)\left(x^{\prime 2}+\right.$ $y^{\prime 2}$ ), where $\Delta$ is the index difference between the center and cladding of the fiber, and $R$ is the fiber core radius. We consider the guiding medium, which corresponds to the case $\Delta>0$.

Equation (1) has a Hamiltonian structure, and it can be rewritten in the dimensionless form by using a change of variables, $\psi=\sqrt{P_{\text {norm }}} A, T=T_{0} t,\left(x^{\prime}, y^{\prime}\right)=r_{0}(x, y), Z=$ $Z_{0} z$, and $\mu=2 \Delta k_{0}^{2} r_{0}^{4} / R^{2}$ [with $\left(\gamma P_{\text {norm }}\right)^{-1}=T_{0}^{2} /\left|\beta_{2}\right|=$
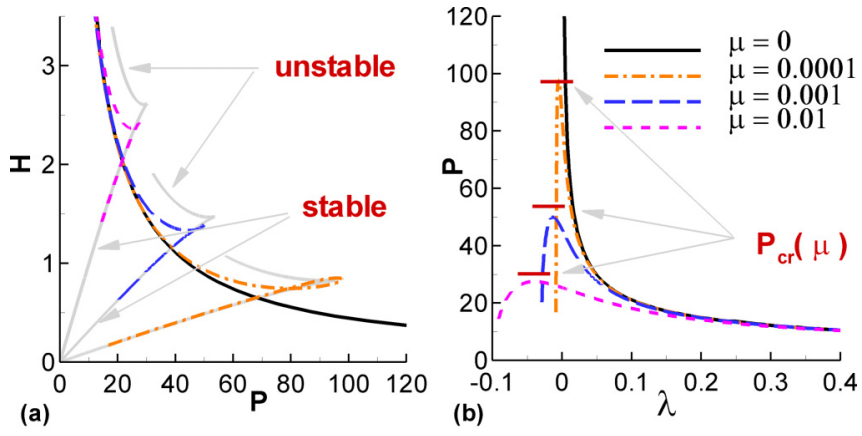

FIG. 2. Families of multidimensional solitons presented through the key dependencies: (a) Hamiltonian $H$ vs power $P$ and (b) power $P$ vs propagation constant $\lambda$. Red bars in (b) show the analytical estimate of the critical power. Gray curves in (a) depict analytical solutions for the fundamental solitons for both stable and unstable branches.

$$
\begin{aligned}
& \left.k_{0} r_{0}^{2}=Z_{0}\right]: \\
& \quad i \frac{\partial A}{\partial z}=\frac{\delta H}{\delta A^{*}}= \\
& -\frac{\sigma}{2} \frac{\partial^{2} A}{\partial t^{2}}-\frac{1}{2}\left(\frac{\partial^{2} A}{\partial x^{2}}+\frac{\partial^{2} A}{\partial y^{2}}\right)+\frac{\mu}{2}\left(x^{2}+y^{2}\right) A-|A|^{2} A,
\end{aligned}
$$

where $\sigma=-\operatorname{sign}\left(\beta_{2}\right)= \pm 1$ (corresponding to the anomalous or normal dispersion, respectively) and the Hamiltonian $H$ is given by the expression

$$
\begin{aligned}
2 H= & \left(\sigma I_{t}+I_{x}+I_{y}+\mu I_{3}-I_{4}\right) \\
= & \int d x d y d t\left[\sigma\left|A_{t}\right|^{2}+\left|A_{x}\right|^{2}+\left|A_{y}\right|^{2}\right. \\
& \left.+\mu\left(x^{2}+y^{2}\right)|A|^{2}-|A|^{4}\right] .
\end{aligned}
$$

Equations (2) and (3) possess several integrals of motion, including Hamiltonian $H$ and power (or the number of particles) $P=\int d t d x d y|A|^{2}$.

\section{MULTIDIMENSIONAL SOLITONS}

We look for steady-state solutions of Eqs. (2) and (3) having the form of spatiotemporal localized modes propagating in the $z$ direction, $A(x, y, z, t)=\exp (i \lambda z) U(x, y, t)$. The waveform of such multidimensional solitons is described by the following equation:

$$
\begin{aligned}
\frac{\delta}{\delta U^{*}}(H+\lambda P)= & 0 \\
= & \lambda U-\frac{\sigma}{2} U_{t t}-\frac{1}{2}\left(U_{x x}+U_{y y}\right) \\
& +\frac{\mu}{2}\left(x^{2}+y^{2}\right) U-|U|^{2} U .
\end{aligned}
$$

This means, in particular, that such solutions should correspond to stationary points of Hamiltonian $H$ for a fixed power $P$.

The resulting steady-state solutions (for $\sigma=1$ ) are the functions of the coordinates $(x, y)$ and time $t$ as well as parameters $\mu$ and $\lambda$. Families of such multidimensional solutions are shown in Figs. $2-5$. 

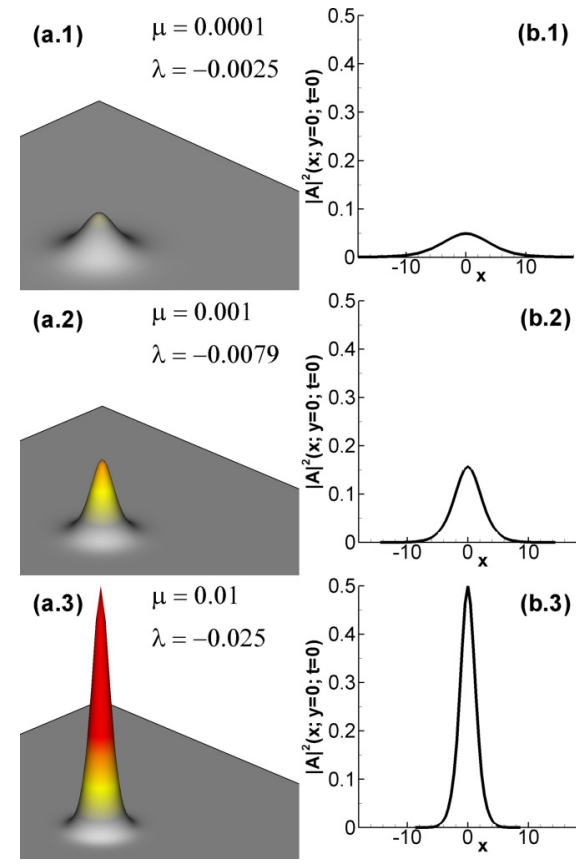

b.3)

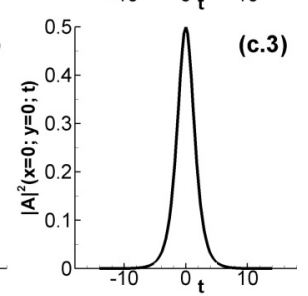

FIG. 3. Examples of three-dimensional solitons, shown for (a.1a.3) power $|A|^{2}(x, y, t)$ in the plane $(x, y)$ for different $\mu$ and $\lambda=$ $-0.25 \sqrt{\mu}$, and power profiles as cross sections in $t$ and $x$.

In Fig. 2(a), we observe how an addition of an external potential creates the second branch of the stable solutions, with a change of the sign of the derivative $d H / d P$ that is a typical signature of the transition from unstable to stable solitons. The same behavior can be traced in Fig. 2(b) where a sign of the derivative $d P / d \lambda$ changes from negative to positive, in accord with the Kolokolov-Vakhitov stability criterion [41]. An approximate analytical stable soliton can be obtained by employing the variational approximation with the Gauss-Hermite trial function provided $E<E_{\mathrm{cr}}=4 \pi \sqrt{\left|\beta_{2}\right| R} /\left(\gamma k_{0} \sqrt[4]{6 \Delta}\right.$ ) (or, in dimensionless units, if $\left.P<P_{c r}(\mu)=4 \pi / \sqrt[4]{3 \mu}\right)$. $E_{\mathrm{cr}}=$ $50 \mathrm{~nJ}$ for a Graded-Index (GRIN) Multimode Fibers. The analytical estimation of the critical power for different values of $\mu$ is marked in Fig. 2(b) by the red bars. Then, the beam width $w_{0}$ and pulse duration $\tau$ are found as

$$
w_{0}^{2}=-\frac{2 R}{k_{0} \sqrt{6 \Delta}} \cos \left(\phi+\frac{2 \pi}{3}\right) \text { and } \tau=\frac{4 \pi\left|\beta_{2}\right| w_{0}^{2}}{\gamma E},
$$

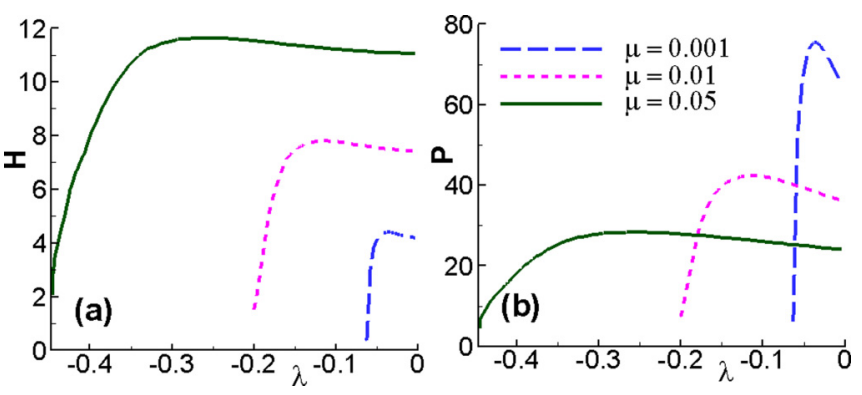

FIG. 4. Families of the dipole-mode spatiotemporal solitons, shown for (a) Hamiltonian $H$ vs propagation constant $\lambda$ and (b) power $P$ vs propagation constant $\lambda$.

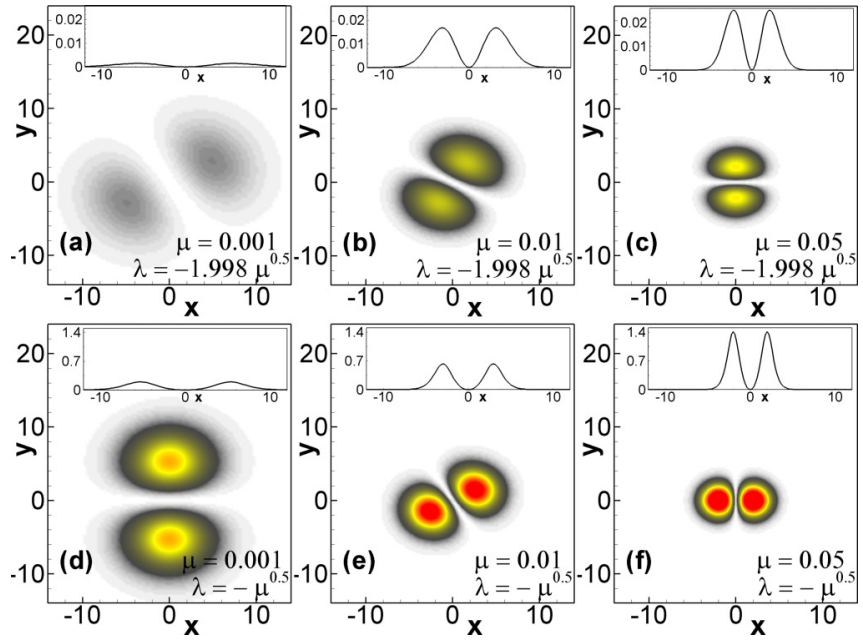

FIG. 5. Power plots and spatial profiles (top) of the dipole-mode multidimensional solitons for different parameters $\mu$ and $\lambda$.

where $\phi=\cos ^{-1}\left(E^{2} / E_{\mathrm{cr}}^{2}\right) / 3$. These theoretical estimations for $w_{0}$ and $\tau$ are valid for the soliton power $E$ below critical. In Fig. 2(a) is shown comparison of the analytical approximation (gray curves) and numerically computed Hamiltonian $H$ versus power $P$.

In addition to the fundamental solitons, we have found solutions with the dipole structure, as shown in Figs. 4 and 5. Again, there are two branches of such solutions with stable and unstable localized modes.

\section{STABILITY AND VARIATIONAL ANALYSIS}

Existence of soliton solutions itself is not sufficient to demonstrate their role in the dynamics of nonlinear systems. The critical issue is stability of these steady-state solutions against perturbations. There are two major approaches to analyze stability of soliton solutions. The first approach is to study the spectrum of linearized operators that describes the evolution of small perturbations of the soliton solution, the stability at the infinitesimal level. The second approach is based on the Lyapunov method, which is a generic technique to analyze stability against perturbations including those that are not necessarily small [3]. In the Hamiltonian systems such as that considered here, according to the Lyapunov theorem (see details in Ref. [3]), a soliton solution is stable provided it corresponds to a minimum of the Hamiltonian. It is well known that in two- and three-dimensional NLSEs solitons are unstable and any initial distribution with high enough power $N$ collapses to a singularity $[3,4]$. In the Hamiltonian systems the classification of a dynamical scenario of a wave is especially transparent. When a Hamiltonian in the considered model is bounded and a solution corresponds to its minimum (or maximum) a corresponding soliton shows up as an attractor. When a Hamiltonian is unbounded, this indicates that soliton solutions correspond to saddle points of the Hamiltonian and are unstable, as for instance in three-dimensional NLSEs. In this case, there is no steady-state asymptotic behavior and either initial wave packets spread out by dispersion (or diffraction, depending of the specific model), effectively demonstrating linear dynamics, or the initial field distribution collapses, that 

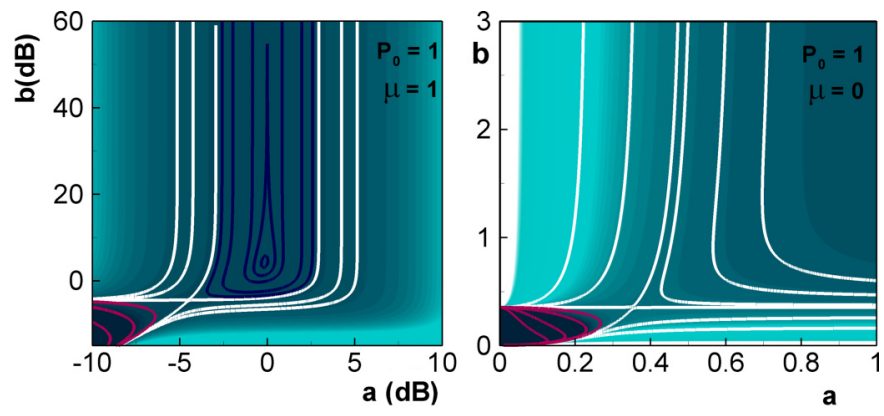

FIG. 6. Two-dimensional Hamiltonian $H(a, b)$ for different values of the parameter $\mu: \mu=1$ (left) and $\mu=0$ (right). Here $\sigma=1$ and $P_{0}=1$. The areas bounded by purple curves correspond to collapse. Blue curves correspond to the dynamics of an input Gaussian pulse corresponding to the fundamental soliton.

mathematically corresponds to formation of a field singularity. Note that for the considered three-dimensional Hamiltonian system, qualitatively, it is clear that at small $\left(x^{2}+y^{2}\right)$ the potential cannot stop collapsing dynamics. There is then an intriguing question, how recently observed stable solitons in multimode fiber coincide with the wave collapse dynamics in such systems. To study this problem, we apply the welldeveloped variational approach that is especially effective for the Hamiltonian systems.

Applying the standard variational approach $[3,4]$ and following an earlier study [42], we analyze the problem of coexistence of stable solitons and wave collapse. The variational approach allows us to obtain a qualitative physical insight, and it is based on presenting Eq. (2) as the variational problem

$$
\delta S=\delta \int d z d t d x d y\left[\frac{i}{2}\left(A^{*} A_{z}-A A_{x}^{*}\right)-H\right]=0
$$

and approximating true solution $A(z, t, x, y)$ by some trial function that mimics the most important properties of the localized mode. We refer to details of the well-known variational approach [3,4,42] and skip mathematical details focusing on new results. We consider a trail function (or the scale transformation) that preserves the total power $P$ :

$$
\begin{aligned}
A(x, y, t, z)= & \frac{\sqrt{P_{0}}}{a(z) b^{1 / 2}(z)} \exp \left[-\frac{x^{2}+y^{2}}{2 a^{2}(z)}-\frac{t^{2}}{2 b^{2}(z)}\right] \\
& \times \exp \left[-i \alpha(z)\left(x^{2}+y^{2}\right)-i \beta(z) t^{2}\right] .
\end{aligned}
$$

Following the standard procedure, we substitute the trial function into the action $S\left[A, A^{*}\right]$ (for details see Refs. [1,3,4,42]) and replace the complex dynamics of waves governed by Eq. (2) by a set of two ordinary differential equations approximating the global dynamics:

$$
\frac{\partial^{2} a}{\partial z^{2}}=-\frac{\partial H(a, b)}{\partial a}, \quad \frac{1}{\sigma} \frac{\partial^{2} b}{\partial z^{2}}=-\frac{\partial H(a, b)}{\partial b} .
$$

Here the effective Hamiltonian becomes a function of the scaling parameters $a$ and $b$ :

$$
H(a, b)=\frac{P_{0} \pi^{3 / 2}}{2}\left(\frac{\sigma}{2 b^{2}}+\frac{1}{a^{2}}+\mu a^{2}-\frac{P_{0}}{2 \sqrt{2} a^{2} b}\right) .
$$

Stable solitons (at $\sigma=1$ ) correspond to minima of the Hamiltonian $H(a, b)$. Straightforward analysis of the extrema points

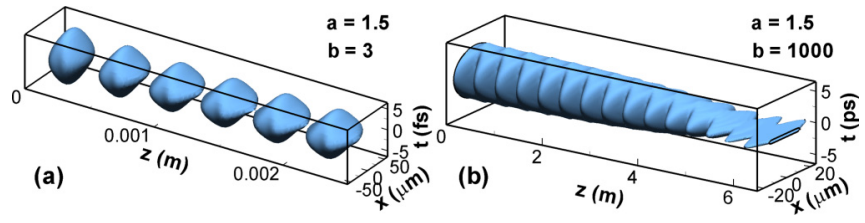

FIG. 7. Results of the direct numerical three-dimensional modeling: $|A|^{2}(x, 0, t, z)$ isosurfaces present (a) the light bullet regime with solitonlike dynamics and (b) the spatiotemporal dynamics with temporal compression.

of $H$ leads to the following condition for the existence of a local minimum of $H$ :

$$
\left(\frac{P_{0}}{4}\right)^{4}<\frac{1}{27 \mu} \text { or }\left(\frac{P}{4}\right)^{4}<\frac{\pi^{6}}{27 \mu} \text {. }
$$

Figure 6 (left) shows the appearance of a local minimum when the existence criterion is satisfied, whereas the right plot represents an unstable case for $\mu=0$. In particular, Fig. 6 (left) shows that, for a signal from the area of minimal $H$ with the power $E=P_{\text {norm }} r_{0}^{2} T_{0} \pi \sqrt{\pi} P_{0} \approx 29 \mathrm{~nJ}$ and nanosecond width, the temporal compression in a GRIN fiber occurs at the width $\tau \approx 3.45 f$ s. Figure 7(b) shows typical corresponding three-dimensional dynamics both in the light bullets regime (a) and in a spatiotemporal dynamics with clear temporal compression.

\section{CONCLUSIONS}

We have analyzed systematically both existence and stability of spatiotemporal solitons in multimode optical fibers, in the framework of the graded-index model. We have revealed that the effective two-dimensional potential formed by the graded refractive index prevents three-dimensional collapse into singularity as is known to occur in uniform three-dimensional media. We have demonstrated the existence of families of stable spatiotemporal solitons and discussed the coexistence of wave collapse and locally stable multidimensional solitons stabilized by the effective low-dimensional parabolic potential in the grade-index multimode fiber, and also found stable dipole-mode spatiotemporal solitons. As a fundamental feature of nonlinear light propagation, these multidimensional solitons might find applications in diverse areas of physics providing new possibilities for control and manipulation of both spatial and temporal properties of light.

\section{ACKNOWLEDGMENTS}

The authors thank L. Berge, D. Christodoulides, A. Rubenchik, and F. Wise for useful discussions and suggestions. The work of O.V.Sh., M.P.F., and S.K.T. was supported by the Russian Science Foundation (Grant No. 17-72-30006). This research was also supported by the Liverhulme Trust (a visiting professorship), the Erasmus Mundus program of the European Union (NanoPhi), and the Australian National University. 
[1] Y. Kivshar and G. Agrawal, Optical Solitons: From Fibers to Photonic Crystals (Academic, New York, 2003).

[2] S. K. Turitsyn, Theor. Math. Phys. 64, 797 (1985).

[3] V. E. Zakharov and E. A. Kuznetsov, Phys. Usp. 55, 535 (2012).

[4] E. A. Kuznetsov, Top. Appl. Phys. 114, 175 (2009); Self-Focusing: Past and Present, edited by R. W. Boyd, S. G. Lukishova, and Y. R. Shen (Springer, New York, 2009).

[5] C. Sulem and P. L. Sulem, Nonlinear Schrodinger Equations: Self-Focusing and Wave Collapse (Springer, New York, 2007).

[6] M. D. Spector, G. E. Fal'kovich, and S. K. Turitsyn, Phys. Lett. A 99, 271 (1983).

[7] E. G. Falkovich and S. K. Turitsyn, Sov. Phys. JETP 62, 146 (1985).

[8] S. K. Turitsyn, Phys. Rev. A 47, R27 (1993).

[9] A. L. Gaeta, Phys. Rev. Lett. 84, 3582 (2000).

[10] L. Bergé and A. Couairon, Phys. Rev. Lett. 86, 1003 (2001).

[11] A. M. Rubenchik, I. S. Chekhovskoy, M. P. Fedoruk, O. V. Shtyrina, and S. K. Turitsyn, Opt. Lett. 40, 721 (2015).

[12] P. M. Lushnikov and N. Vladimirova, Opt. Express 23, 31120 (2015).

[13] V. V. Flambaum and E. A. Kuznetsov, Nonlinear dynamics of ultra-cold gases: Collapse of Bose gas with attractive interaction, in Proceedings of NATO Advanced Research Workshop on Singularities in Fluids, Plasmas and Optics (Heraklion, Greece), edited by R. E. Caflisch and G. C. Papanicolaou (Kluwer Academic, Dordrecht, The Netherlands, 1992).

[14] Yu. S. Kivshar and D. E. Pelinovsky, Phys. Rep. 331, 117 (2000).

[15] X. Liu, L. J. Qian, and F. W. Wise, Phys. Rev. Lett. 82, 4631 (1999).

[16] S. Minardi, F. Eilenberger, Y. V. Kartashov, A. Szameit, U. Röpke, J. Kobelke, K. Schuster, H. Bartelt, S. Nolte, L. Torner, F. Lederer, A. Tünnermann, and T. Pertsch, Phys. Rev. Lett. 105, 263901 (2010).

[17] F. Eilenberger, K. Prater, S. Minardi, R. Geiss, U. Ropke, J. Kobelke, K. Schuster, H. Bartelt, S. Nolte, A. Tunnermann, and T. Pertsch, Phys. Rev. X 3, 041031 (2013).

[18] P. J. Winzer, IEEE Photonics J. 4, 647 (2012).

[19] D. J. Rochardson, J. M. Fini, and L. E. Nelson, Nat. Photonics 7, 354 (2013).

[20] W. H. Renninger and F. W. Wise, Nat. Commun. 4, 1719 (2013).
[21] A. Hasegawa, Opt. Lett. 5, 416 (1980).

[22] B. Crosignani and P. D. Porto, Opt. Lett. 6, 329 (1981).

[23] B. A. Malomed, D. Mihalache, F. Wise, and L. Torner, J. Opt. B 7, R53 (2005).

[24] A. Mafi, J. Lightwave Technol. 30, 2803 (2012).

[25] L. G. Wright, D. N. Christodoulides, and F. W. Wise, Nat. Photonics 9, 306 (2015).

[26] S. Buch and G. P. Agrawal, Opt. Lett. 40, 225 (2015).

[27] L. G. Wright, W. H. Renninger, D. N. Christodoulides, and F. W. Wise, Opt. Express 23, 3492 (2015).

[28] L. G. Wright, S. Wabnitz, D. N. Christodoulides, and F. W. Wise, Phys. Rev. Lett. 115, 223902 (2015).

[29] K. Krupa, A. Tonello, A. Barthelemy, V. Couderc, B. M. Shalaby, A. Bendahmane, G. Millot, and S. Wabnitz, Phys. Rev. Lett. 116, 183901 (2016).

[30] L. Salasnich, A. Parola, and L. Reatto, Phys. Rev. A 66, 043603 (2002)

[31] B. B. Baizakov, B. A. Malomed, and M. Salerno, Phys. Rev A 70, 053613 (2004).

[32] E. V. Shuryak, Phys. Rev. A 54, 3151 (1996).

[33] C. A. Sackett, H. T. C. Stoof, and R. G. Hulet, Phys. Rev. Lett. 80, 2031 (1998).

[34] J. M. Gerton, D. Strekalov, I. Prodan, and R. G. Hulet, Nature (London) 408, 692 (2000).

[35] L. Bergé, T. J. Alexander, and Yu. S. Kivshar, Phys. Rev. A 62 , 023607 (2000).

[36] T. Tsurumi, H. Morise, and M. Wadati, Int. J. Mod. Phys. B 14, 655 (2000); H. Morise and M. Wadati, J. Phys. Soc. Jpn. 70, 3529 (2001).

[37] A. V. Rybin, G. G. Varzugin, M. Lindberg, J. Timonen, and R. K. Bullough, Phys. Rev. E 62, 6224 (2000).

[38] B. J. Cusack, T. J. Alexander, E. A. Ostrovskaya, and Y. S. Kivshar, Phys. Rev. A 65, 013609 (2001).

[39] D. Buccoliero, A. S. Desyatnikov, W. Krolikowski, and Yu. S. Kivshar, Phys. Rev. Lett. 98, 053901 (2007).

[40] J. Carpenter, B. J. Eggleton, and J. Schröder, Nat. Photonics 9 , 751 (2015).

[41] A. A. Kolokolov and N. B. Vakhitov, Radiophys. Quantum Electron. 16, 783 (1973).

[42] S. Raghavan and G. P. Agrawal, Opt. Commun. 180, 377 (2000). 